\title{
Knockdown of Med19 Suppresses Proliferation and Enhances Chemo-sensitivity to Cisplatin in Non-small Cell Lung Cancer Cells
}

\author{
Ling Wei ${ }^{1}$, Xing-Wu Wang ${ }^{1}$, Ju-Jie Sun ${ }^{2}$, Li-Yan Lv ${ }^{1}$, Li Xie ${ }^{1}$, Xian-Rang Song ${ }^{1 *}$
}

\begin{abstract}
Mediator 19 (Med19) is a component of the mediator complex which is a coactivator for DNA-binding factors that activate transcription via RNA polymerase II. Accumulating evidence has shown that Med19 plays important roles in cancer cell proliferation and tumorigenesis. The involvement of Med19 in sensitivity to the chemotherapeutic agent cisplatin was here investigated. We employed RNA interference to reduce Med19 expression in human non-small cell lung cancer (NSCLC) cell lines and analyzed their phenotypic changes. The results showed that after Med19 siRNA transfection, expression of Med19 mRNA and protein was dramatically reduced $(p<0.05)$. Meanwhile, impaired growth potential, arrested cell cycle at G0/G1 phase and enhanced sensitivity to cisplatin were exhibited. Apoptosis and caspase-3 activity were increased when cells were exposed to Med19 siRNA and/or cisplatin. The present findings suggest that Med19 facilitates tumorigenic properties of NSCLC cells and knockdown of Med19 may be a rational therapeutic tool for lung cancer cisplatin sensitization.
\end{abstract}

Keywords: Med19 - non-small cell lung cancer - sensitivity -cisplatin - RNAi

Asian Pac J Cancer Prev, 16 (3), 875-880

\section{Introduction}

Non-small cell lung cancer (NSCLC), a malignant tumor type with a high rate of relapse and metastasis, is the leading cause of cancer-related deaths worldwide. Despite aggressive approaches to treatment of lung cancer, the prognosis of NSCLC patients is still poor, with a 5-year survival rate of 5-14\% (Khuri et al., 2001). This is usually due to the fact that most patients with this disease receive a late diagnosis and experience a relapse because of the chemoresistance (Rosell et al., 2006; Jang et al., 2009). Therefore, there is an urgent need to identify novel prognostic marker and therapeutic target for NSCLC.

The mediator (Med) complex, an essential component of the RNA polymerase II ( pol II)-mediated transcription complex, plays a crucial role in the stimulation of basal transcription and regulation of eukaryotic mRNA synthesis, which underpins cell proliferation, development, differentiation, and maintenance (Conaway et al., 2005; Conaway et al., 2005; Casamassimi et al., 2007). Med is a crescent-shaped complex consisting of head, middle, tail modules and highly conserved from yeast to mammals in evolution (Boube et al., 2002). As a multi-protein coactivator, Med consists of about 30 subunits that can interact with transcriptional activators, such as p53, SP1 and VP16 (Malik et al., 2005; Vojnic et al., 2011). Human Med19 gene, also known as "lung cancer metastasis related protein 1" (LCMR1), is located on human chromosome 11 at the 11q12.1 locus and involved in the head-module subunits of Mediator. Recently, accumulating evidence has shown that Med19 is associated with cancer cell proliferation, tumorigenesis and prognosis, suggesting that Med19 may be an attractive candidate for the therapeutic target of cancer (Chen et al., 2011a; Cui et al., 2011a; Li et al., 2011a; Sun et al., 2011a; Wang et al., 2011a; Zou et al., 2011a; Ji-Fu et al., 2012a; Zhang et al., 2012a; Zhu et al., 2013a; Yu et al., 2014a). In human NSCLC, overexpression of Med19 significantly associated with clinical stage and pathogenesis, indicating that Med19 may have applications in the clinical diagnosis and treatment of lung cancer (Chen et al., 2011b). However, whether Med19 is involved in chemoresistance to cisplatin, a commonly used chemotherapeutic agent in NSCLC treatment, remains largely unknown.

In the present study, we employed siRNA to inhibit the expression of Med19 in NSCLC cells and analyze the biological behaviors. We report for the first time that silencing of Med19 can enhance cisplatin chemosensitivity and apoptosis in NSCLC cell lines. Caspase-3 activity and apoptosis were induced when cells were exposed to Med19 siRNA and/or cisplatin. Our results thus suggest that Med19 inhibition could be a promising approach for chemo-sensitization of NSCLC cells to applied cisplatin therapy. 


\section{Materials and Methods}

\section{Cell culture}

Human lung adenocarcinoma cell lines, SPCA1 and A549, were obtained from China Centre for Type Culture Collection (CCTCC, Wuhan, China) and American Type Culture Collection (ATCC, Manassas, VA, USA), respectively. The cells were grown in Dulbecco's modified Eagle's medium (DMEM, Gibco) containing 10\% fetal bovine serum (FBS, Gibco), 100 units/mL penicillin and $100 \mu \mathrm{g} / \mathrm{ml}$ streptomycin at $37^{\circ} \mathrm{C}$ in a humidified atmosphere of $5 \% \mathrm{CO}_{2}$.

\section{Med19 siRNA transfection}

Silencing of Med19 expression was obtained through transfection of med19 siRNA. SiRNA targeting the sequence of human Med19 gene (NM_153450.1) is 5'-GGTGAAGGAGAAGCTAAGT-3', designed with an online software of Invitrogen and has been evidenced to be effective in many reports (Cui et al., 2011b; Li et al., 2011b; Zou et al., 2011b; Li et al., 2011b; Sun et al., 2011b; Wang et al., 2011b; Zhang et al., 2012b; Ji-Fu et al., 2012b; $\mathrm{Yu}$ et al., 2014). The sequences for the control siRNA were sense: 5'-UUCUCCGAACGUGUCACGUTT-3'; antisense: 5'-ACGUGACACGUUCGGAGAATT-3'. The indicated siRNA were synthesized by Genepharma (Shanghai, China). Cell transfection was performed using Lipofectamine $^{\mathrm{TM}} 2000$ reagent (Invitrogen, Carlsbad, CA, USA) according to the manufacturer's instructions. Briefly, NSCLC cells were seeded in six-well plates at a density of (2-3) $\times 10^{5}$ cells/well and grown overnight. Then, the cells were transfected for the indicated time under standard culture conditions with $100 \mathrm{nM}$ siRNA duplexes using Lipofectamine ${ }^{\mathrm{TM}} 2000$ reagent.

\section{Real-time RT-PCR analysis}

For real-time RT-PCR analysis, total cellular RNA was extracted with Trizol (Invitrogen). Reverse transcription was performed by Super-Script II reverse transcriptase (TaKaRa code DRR037A, Dalian, China). The Med19 and internal control $\beta$-actin gene were amplified with SYBR $^{\circledR}$ Premix Ex Taq ${ }^{\text {TM }}$ quantitative PCR kit (Takara code DRR041A, Dalian, China). Primers for Med19 (forward 5'-TGACAGGCAGCACGAATC-3'; reverse 5'-CAGGTCAGGCAGGAAGTTAC-3') and $\beta$-actin (forward 5'-TTAGTTGCGTTACACCCTTTC-3'; reverse 5'-GCTGTCACCTTCACCGTTC-3') were synthesized by Sangon (Shanghai, China). Relative expression of Med19 mRNA was calculated using the comparative CT method.

\section{Immunoblot analysis}

The indicated cells were lysed by precooled lysis buffer containing $0.15 \mathrm{M} \mathrm{NaCl}, 50 \mathrm{mM}$ Tris- $\mathrm{Cl}$ (pH 7.4), $2 \mathrm{mM}$ EDTA (pH 8.0), 0. 5\% Triton-100, 5 mM DTT, $0.2 \mathrm{mM}$ PMSF and $2 \mu \mathrm{g} / \mathrm{ml}$ aprotinin. Bradford method was applied to determine the protein content. $50 \mu \mathrm{g}$ total protein lysates were separated by $10 \%$ SDS-polyacrylamide gels and the gels were subjected to immunoblotting. The resulting PVDF membrane was blocked in TBST (10 mM Tris [pH 8.0], $150 \mathrm{mM} \mathrm{NaCl}, 0.05 \%$ Tween-20) blocking buffer containing 5\% non-fat dry milk. The primary antibodies, rabbit anti-human Med19 (Abcam, Cambridge, UK) and mouse anti-human $\beta$-actin (Santa Cruz Biotechnology, Santa Cruz, CA) antibodies were added at a ratio of 1: 400 and 1: 2000, respectively, and incubated overnight at $4^{\circ} \mathrm{C}$. The membrane was then washed thrice with TBST, followed by incubation for $1 \mathrm{~h}$ with appropriate secondary antibody coupled to horseradish peroxidase (Santa Cruz Biotechnology) at room temperature. After further washing, enhanced chemiluminescence (ECL plus, Amersham Pharmacia Biotech) reagents were used to detect the signals, according to the manufacturer's instructions.

\section{Cell cycle analysis}

To determine whether Med 19 silencing affected cell cycle progression, NSCLC cells were transfected with Med19 siRNA for $72 \mathrm{~h}$. Then, the indicated cells were fixed in $70 \%$ ethanol at $4^{\circ} \mathrm{C}$ overnight. Next, cells were washed twice with cold PBS and stained with a solution containing $50 \mu \mathrm{g} / \mathrm{ml}$ propidium iodide (PI) and 100 $\mathrm{U} / \mathrm{ml}$ RNase A in PBS for $30 \mathrm{~min}$ in the dark at room temperature. Flow cytometric analysis was performed on a FACS Calibur flow cytometer (BD Bioscience, CA).

\section{Cell proliferation assay}

For determination of cell growth, [3-(4,5-dimethyl-2thiazolyl)-2,5-diphenyl-2H-tetrazolium bromide (MTT) assay was used. Cells transfected with Med19 siRNA for $48 \mathrm{~h}$ were seeded in 96-well plates at a density of 3000 cells per well. After incubation for 24, 48, 72 and 96h, 20 $\mu \mathrm{L}$ of MTT $(5 \mathrm{mg} / \mathrm{mL})$ was added. After $4 \mathrm{~h}$ of incubation, $100 \mu \mathrm{L}$ of $10 \%$ dimethyl sulfoxide (SDS) containing $0.01 \mathrm{~N} \mathrm{HCL}$ was added to each well. After being incubated for $24 \mathrm{~h}$ at $37^{\circ} \mathrm{C}$, the absorbance was measured at $570 \mathrm{~nm}$ with a microplate reader (Bio-rad 680, USA).

\section{Colony formation assay}

Cells transfected with Med19 siRNA for 48 h were seeded in 6-well plates with 200 cells per well and cultured at $37^{\circ} \mathrm{C}$ for 14 days. At the end of incubation, the cells were washed with PBS twice and fixed with methanol/acetic acid (75\%: 25\%) and stained with crystal violet. Visible colonies (containing 50 or more cells each) were counted. All clonogenic assays were repeated as independent experiments at least twice.

\section{Cisplatin sensitivity evaluation via MTT assay}

After transfected with Med19 siRNA for $48 \mathrm{~h}$, the indicated cells were trypsinized and plated into 96-well plates at a density of $10^{4}$ cells/well, followed by treatment with the indicated doses of cisplatin $(0,0.625,1.25,2.5,5$, $10 \mu \mathrm{g} / \mathrm{ml}$ ) for $24 \mathrm{~h}$. Then, cells were incubated with $20 \mu \mathrm{L}$ of MTT $(5 \mathrm{mg} / \mathrm{ml})$ for $4 \mathrm{~h}$ at $37^{\circ} \mathrm{C}$. Finally, the resulting formazan crystals were solubilized with $100 \mu \mathrm{L}$ of $10 \%$ SDS containing $0.01 \mathrm{~N}$ HCL. Absorbance was measured at $570 \mathrm{~nm}$ by a microplate reader (Bio-Rad 680, USA), and the cell viability was calculated.

Determination of apoptosis by flow cytometry

To determine the effect of Med19 depletion on 
apoptosis, NSCLC cells were transfected with Med 19 siRNA for $48 \mathrm{~h}$, followed by exposure to the indicated concentration of cisplatin $(2 \mu \mathrm{g} / \mathrm{ml})$ for $24 \mathrm{~h}$. Subsequently, cells were stained with Annexin V-FITC Apoptosis Kit (BD Pharmingen, CA) and subjected to FACS Calibur flow cytometer.

\section{Caspase-3 activity assay}

A caspase- 3 activity assay kit (Beyotime Institute of Biotechnology, Haimen, China) was used to evaluate the caspase-3 activity of lung cancer cells, according to the manufacturer's protocols. After treatment with cisplatin for $24 \mathrm{~h}$, the indicated cells were washed with ice-cold PBS and homogenized in lysis buffer. Lysates were centrifuged at $16,000 \mathrm{~g}$ for $10 \mathrm{~min}$, and Bradford protein assay was used to determine protein concentrations. $30 \mu \mathrm{g}$ of protein was incubated with $10 \mu \mathrm{l}$ of Ac-DEVD-pNA at $37^{\circ} \mathrm{C}$ for $4 \mathrm{~h}$ in a $96-w e l l$ microtiter plate. Samples were measured with an ELISA reader at an absorbance of $405 \mathrm{~nm}$.

\section{Statistical analysis}

Data were presented as mean \pm SD. SPSS 13.0 software (SPSS, Chicago, USA) was used to perform statistical analysis. Statistical significance was set at $p<0.05$.

\section{Results}

Med19 siRNA specifically inhibits the expression of Med19 $m R N A$ and protein in lung cancer cells

Real-time RT-PCR was applied to assess the Med19 mRNA expression in parental cells, control or Med19 siRNA transefected NSCLC cells. As shown in Figure 1A, the expression of Med19 mRNA in Med19 siRNA group were significantly down-regulated compared with control siRNA group $(p<0.05)$. No statistical significances were observed between control siRNA and parental cells $(p>0.05)$. Moreover, Med19 siRNA transfected cells exhibited significant reduced levels of Med19 protein compared with control siRNA and parental cells $(p<0.05$; Figure 1B). At the same time, another Med19 siRNA also demonstrated similar RNAi efficacy (data not shown). The sequences for this Med19 siRNA were as follows: sense: 5'-CGAAUCUGAUCACACACUATT -3'; antisense: 5'- UAGUGUGUGAUCAGAUUCGTT-3'.

Med19 knockdown leads to impaired cell proliferation and colony formation of NSCLC cells

Two days after transfection, all three groups of SPCA1 and A549 cells were plated for MTT and colony formation assays to observe cell growth. Compared with control siRNA, the proliferation of Med19 siRNA transfected cells were dramatically suppressed (Figure 2A). In colony formation assay, similar results were obtained. Both NSCLC cells exhibited reduced colony formation potentials in Med19 siRNA cells (Figure 2B). These findings indicate that knockdown of endogenous Med19 inhibited the proliferation of NSCLC cells.

Knockdown of Med19 induces apoptosis and regulates cell cycle progression

In order to evaluate the effect of Med19 on apoptosis and cell cycle distribution, flow cytometry assay was performed. As shown in Figure 3A, Med19 silencing increased apoptosis compared with control siRNA group $(p<0.05)$, indicating that Med19 influenced the process of apoptosis. In the profile of cell cycle distribution, the percentage of G0/G1 phase was increased in Med19 siRNA treated cells (SPCA1, control vs. Med19 siRNA, $67.8 \pm 3.8$ vs $75.8 \pm 4.3, p<0.05$; A549, control vs. Med19 siRNA, $59.5 \pm 3.3$ vs $67.2 \pm 3.6, p<0.05)$, whereas the percentage of $\mathrm{S}$ phase was decreased in these cells compared with control siRNA cells (SPCA1, control vs.

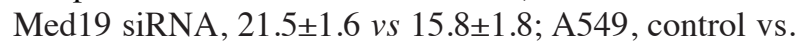
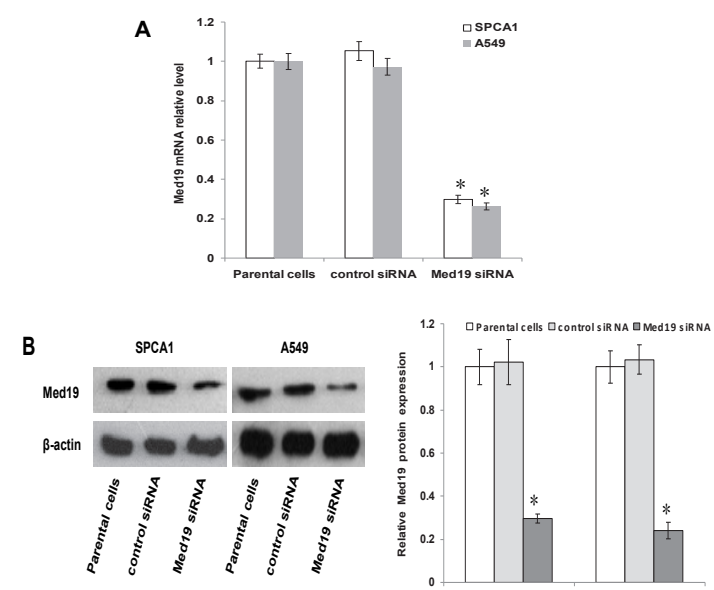

Figure 1. Med19 siRNA Mediated RNAi Dramatically Suppressed Med19 Expression in Lung Cancer Cells. (A) Expression of Med19 mRNA detected by real-time RTPCR. Compared with Parental cells and control siRNA group, Med19 mRNA expression dramatically decreased in Med19 siRNA group. ${ }^{*} \mathrm{P}<0.05$ : Med19 siRNA versus control siRNA; (B) Immunoblot analysis of Med 19 protein expression. $\beta$-actin was used to an internal control. Med19 protein expression was significantly lower in Med19 siRNA group. ${ }^{*} p<0.05$ : Med19 siRNA versus control siRNA
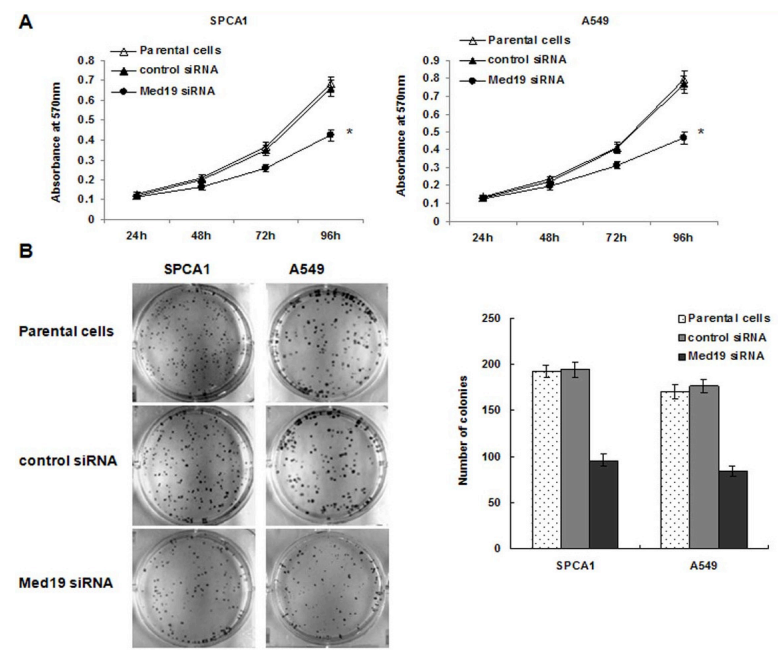

Figure 2. Knockdown of Med19 Attenuated Cell Growth of SPCA1 and A549 Cells. (A) Influence of Med19 knockdown on proliferation of SPCA1 and A549 cells were detected by the MTT assay. ${ }^{*} p<0.05$ : Med19 siRNA versus control siRNA. (B) Effect of Med19 knockdown on growth of SPCA1 and A549 cells were detected by the colony formation assay. ${ }^{*} p<0.05$ : Med19 siRNA versus control siRNA 


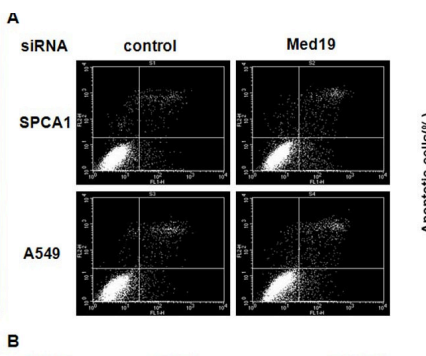

B

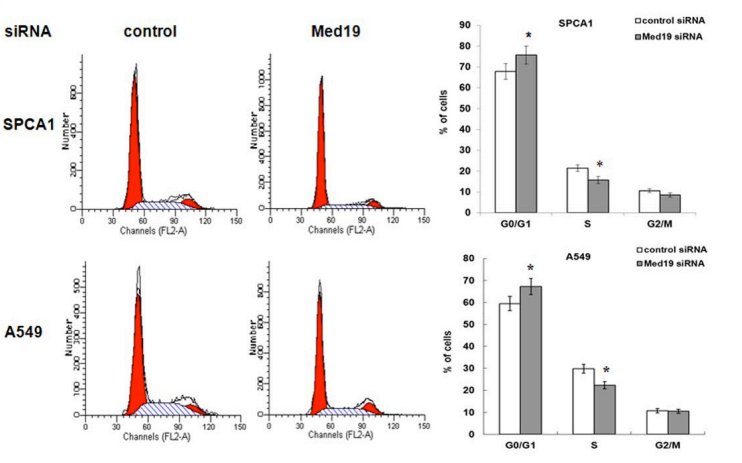

Figure 3. Knockdown of Med19 Expression Induced Apoptosis and Regulated Cell Cycle Progression. (A) Med19 siRNA transfected cells were subjected to Annexin V-FITC and PI apoptosis analysis. The rate of apoptosis was higher in Med19 siRNA cells than that in control siRNA cells. *P<0.05: Med19 siRNA versus control siRNA; (B) Cell cycle distribution was detected by PI staining followed by flow cytometric analysis. The proportions at G0/G1 and S phases were significantly increased and reduced, respectively, in Med19 siRNA cells than that in control siRNA cells. ${ }^{*} p<0.05$ : Med19 siRNA versus control siRNA

Med19 siRNA, 29.8 \pm 2.0 vs 22.3 $\pm 1.7, p<0.05$ ) (Figure $3 B)$. These results indicated that Med19 regulated cell cycle progression at the G1/S boundary.

Med19 silencing increases cisplatin sensitivity, cisplatininduced apoptosis and caspase-3 activity in NSCLC cells

To determine the effect of Med19 silencing on cisplatin sensitivity of NSCLC cells, the indicated cells were treated with various concentrations of cisplatin for $24 \mathrm{~h}$. Our results showed that the viability of Med19 silenced cells were more decreased than that of control siRNA treated cells at the same concentration of cisplatin (Figure 4A), indicating that Med19 inhibition may enhance the sensitivity to cisplatin in NSCLC cells. To explore the effect of Med19 silencing on cisplatin-induced apoptosis, we detected apoptosis using a standard Annexin V assay. A significant increase in cisplatin-induced apoptosis was observed in cells transfected with Med19 siRNA, compared with control siRNA transfected cells (Figure 4B and 4C). These results revealed that Med19 depletion potentiates cisplatin sensitivity and promotes cisplatininduced apoptosis. As caspase- 3 is a critical enzyme of the execution phase of apoptosis, we investigated the influence of Med19 silencing on caspase-3 activity. Data showed that caspase- 3 is highly activated in Med19 siRNA treated cells compared with the control cells after cisplatin treatment (Figure 4D).

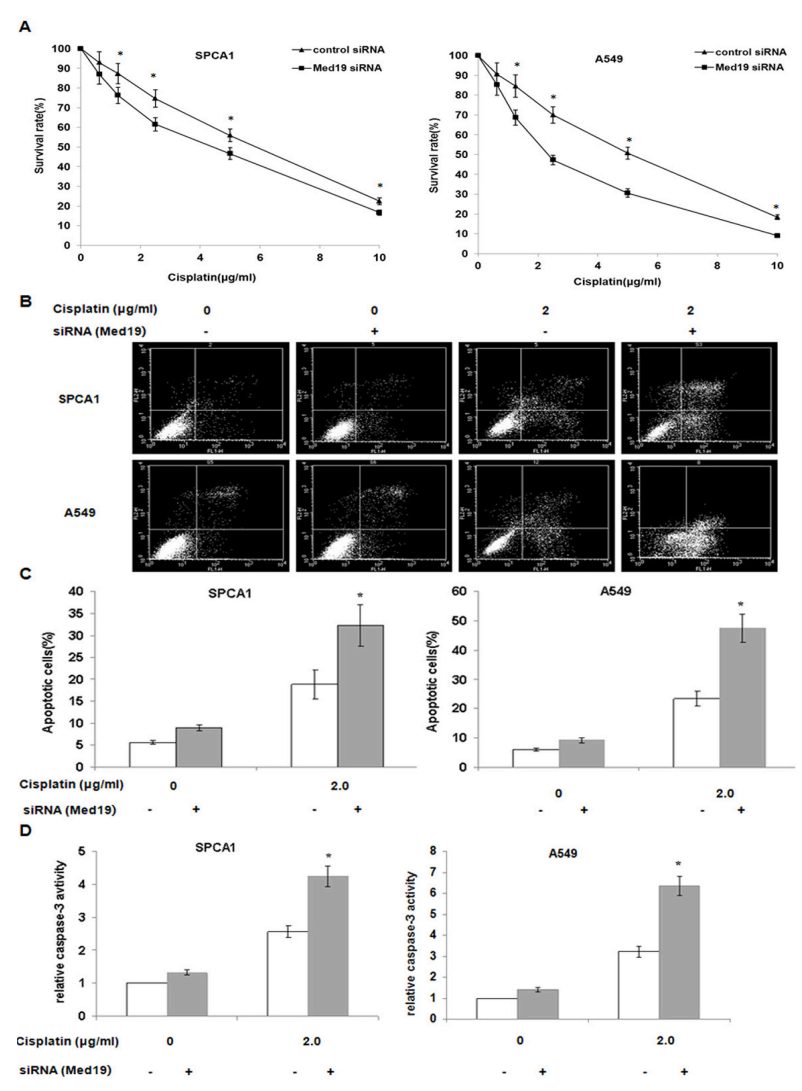

Figure 4. Med19 Knockdown Increases Cisplatin Sensitivity, Cisplatin-Induced Apoptosis and Caspase-3 Activity in NSCLC Cells. SPCA1 and A549 cells were transfected with Med19 siRNA for $48 \mathrm{~h}$, then treated with cisplatin for 24h. (A) MTT assay was used to determine the viability. The percentage of cell growth was calculated by comparison of the A570 reading from treated cells versus control cells. ${ }^{*} p<0.05$ : Med19 siRNA versus control siRNA. (B) Apoptosis was determined using Annexin V-FITC plus PI staining. Representative data are shown. (C) Population of apoptosis was counted. Similar results were obtained in three separate experiments. Columns, mean; bars, s.d. $(n=3)$. ${ }^{*} p<0.05$ : Med19 siRNA versus control siRNA. (D) The activity of caspase- 3 following cisplatin treatment augmented in Med19 silencing cells compared with control siRNA cells. ${ }^{*} p<0.05$ : Med19 siRNA versus control siRNA

\section{Discussion}

Emerging evidence has demonstrated that Med19 is a novel proliferation regulator that promotes cancer cells growth and tumorigenesis, including cancers of breast, bladder, colorectal and lung (Sun et al., 2011c; Li et al., 2011c; Zou et al., 2011c; Xu et al., 2012; Zhang et al., 2012c; Ji-Fu et al., 2012c; Zhu et al., 2013b; Wen et al., 2013). Recently, it was reported that Med19 was strongly overexpressed in cancer tissues of NSCLC patients and the expression was significantly associated with clinical stage (Chen et al., 2011c). However, the detailed biological effects of Med19 on NSCLC, especially the relation between Med19 and chemosensitivity to cisplatin, has not been clarified.

In this study, we disclosed that suppression of Med19 
results in impaired potentials of proliferation and colony formation in NSCLC cells (Figure 1), indicating that Med19 plays an important role in cell growth. Similar with the results from cells of breast cancer and hepatocellular carcinoma (Zou et al., 2011d; Zhang et al., 2012d). We also found that Med19 down-regulation elicited augmentation of G0/G1 phase proportion, showing that Med19 may be involved in control of post-mitotic cellular processe in NSCLC. The G1 checkpoint is controlled by cyclin D, p21 and CDK4/6 (Satyanarayana et al., 2008; Wang et al., 2012). Whether Med19 contributing to the activity of CDK4/6-Cyclin D complexes in NSCLC cells needs to be further elucidated.

Chemotherapy based on cisplatin, the most widely used regimen in NSCLC first-line chemotherapy, plays a crucial role in NSCLC comprehensive treatment. However, inherent and acquired drug resistance limited therapeutic efficacy. It has been shown that cisplatin resistance is associated with multifactorial mechanisms, such as inactivation by glutathione, metallothionein, or other sulphur-containing molecules, increased repair of DNA damage and Autophagy, reduced apoptosis and intracellular drug accumulation by changing the profile of drug-influx or -efflux molecules (Song et al., 2012; Galluzzi et al., 2012; Zhang et al., 2013). Many studies, concerned with the specific gene manipulation, have provided a way to make cancer cells specifically more sensitive to cisplatin (Hu et al., 2012; Li et al., 2012; Yu et al., 2013). However, the role of Med19 in chemotherapeutic drug resistance has not yet been established. This work was the first study to demonstrate that Med19 silencing enhances chemosensitivity to cisplatin and sensitizes NSCLC cells to cisplatin-induced apoptosis, indicating that Med19 inhibition is applicable to developing combination chemotherapy of cisplatin.

Activation of caspase- 3 is a key and common event of two major apoptosis signaling pathways, mitochondrial pathway and death receptor pathway (Mazumder et al., 2008; Snigdha et al., 2012). The death receptor pathway activates initiator caspase- 8 and then cleaves its downstream effector caspases, such as caspase- 3 which transducts the apoptosis signal, while the mitochondrial pathway is initiated by the mitochondrial dysfunction and then causes activation of caspase- 9 which triggers caspase-3 activation (Fulda et al., 2006). Studies have shown that Med19 knockdown results in the upregulation of Bax and downregula $\neg$ tion of Mcl-1 (Xu et al., 2012). In human prostate cancer cells PC-3 and DU145, knockdown of Med19 induced apoptosis via modulation of Bid and Caspase 7 (Cui et al., 2011c). Recent evidence has shown that Med19 knockdown can induce apoptosis in osteosarcoma celll line Saos-2 via induction of caspase-3 and poly ADP-ribose polymerase (PARP), along with the downregulated expression of cyclin D1 and cyclin B1 (Yu et al., 2014). Inhibition of cyclin D1 enhanced cisplatin sensitivity have been documented in human breast cancer MCF-7 and human oral squamous cell carcinoma cells. The present data demonstrated that combination of Med19 siRNA and cisplatin led to caspase- 3 catalytic activity in NSCLC cells to a level that was dramatically higher than that observed with cisplatin alone. Apoptotic pathway and related protein molecules involved in Med19 silencing enhancing cisplatin-induced apoptosis and sensitivity of NSCLC cells need to be further investigated.

Taken together, the present data has identified that siRNA-mediated Med19 down-regulation attenuates the carcinogenic properties of NSCLC cells. To the best of our knowledge, this is the first report to present a siRNA-mediated Med19 silencing strategy to potentiate chemosensitivity and apoptosis induced by cisplatin. These findings indicate that combination of Med19 down-regulation and cisplatin may be a new therapeutic strategy for NSCLC. However, this study has limitations. We only focused on a chemotherapeutic drug cisplatin, further investigation on other chemotherapeutic agents might strengthen the significance of our study.

\section{Acknowledgements}

This research is supported by grants from Shandong Province Natural Science Foundation (ZR2009CM141, ZR2010HM083) and Shandong Health Department (2011HZ095), China.

\section{References}

Boube M, Joulia L, Cribbs DL, et al (2002). Evidence for a Mediator of RNA polymerase II transcriptional regulation conserved from yeast to man. Cell, 110, 143-51.

Casamassimi A, Napoli C (2007). Mediator complexes and eukaryotic transcription regulation: an overview. Biochimie, 89, 1439-46.

Chen L, Liang Z, Tian Q, et al (2011). Overexpression of LCMR1 is significantly associated with clinical stage in human NSCLC. J Exp Clin Cancer Res, 30, 18.

Conaway JW, Florens L, Sato S, et al (2005). The mammalian Mediator complex. FEBS Lett, 579, 904-8.

Conaway RC, Sato S, Tomomori-Sato C, et al (2005). The mammalian Mediator complex and its role in transcriptional regulation. Trends Biochem Sci, 30, 250-5.

Cui X, Xu D, Lv C, et al (2011). Suppression of MED19 expression by shRNA induces inhibition of cell proliferation and tumorigenesis in human prostate cancer cells. $B M B$ Rep, 44, 547-52.

Fulda S, Debatin KM (2006). Extrinsic versus intrinsic apoptosis pathways in anticancer chemotherapy. Oncogene, 25, 4798811.

Galluzzi L, Senovilla L, Vitale I, et al (2012). Molecular mechanisms of cisplatin resistance. Oncogene, 31, 1869-83.

Jang RW, Le Maître A, Ding K, et al (2009). Quality-adjusted time without symptoms or toxicity analysis of adjuvant chemotherapy in non-small-cell lung cancer: an analysis of the national cancer institute of Canada clinical trials group JBR.10 trial. J Clin Oncol, 27, 4268-73.

Ji-Fu E, Xing JJ, Hao LQ, et al (2012). Suppression of lung cancer metastasis-related protein 1 (LCMR1) inhibits the growth of colorectal cancer cells. Mol Biol Rep, 39, 3675-81.

$\mathrm{Hu} \mathrm{MD}$, Xu JC, Fan Y, et al (2012). Hypoxia-inducible factor 1 promoter-induced JAB 1 overexpression enhances chemotherapeutic sensitivity of lung cancer cell line A549 in an anoxic environment. Asian Pac J Cancer Prev, 13, 2115-20.

Khuri FR, Herbst RS, Fossella FV (2001). Emerging therapies in non-small-cell lung cancer. Ann Oncol, 12, 739-44.

Li HL, Huang DZ, Deng T, et al (2012). Overexpression of cyclin L2 inhibits growth and enhances chemosensitivity in human 
gastric cancer cells. Asian Pac J Cancer Prev, 13, 1425-30.

Li LH, He J, Hua D, et al (2011). Lentivirus-mediated inhibition of Med19 suppresses growth of breast cancer cells in vitro. Cancer Chemother Pharmacol, 68, 207-15.

Li XH, Fang DN, Zeng CM (2011). Knockdown of MED19 by short hairpin RNA-mediated gene silencing inhibits pancreatic cancer cell proliferation. Cancer Biother Radiopharm, 26, 495-501.

Malik S, Roeder RG (2005). Dynamic regulation of pol II transcription by the mammalian Mediator complex. Trends Biochem Sci, 30, 256-63.

Mazumder S, Plesca D, Almasan A (2008). Caspase-3 activation is a critical determinant of genotoxic stress-induced apoptosis. Methods Mol Biol, 414, 13-21.

Rosell R, Cecere F, Santarpia M, et al (2006). Predicting the outcome of chemotherapy for lung cancer. Curr Opin Pharmacol, 6, 323-31.

Satyanarayana A, Hilton MB, Kaldis P(2008). p21 Inhibits Cdk1 in the absence of $\mathrm{Cdk} 2$ to maintain the G1/S phase DNA damage checkpoint. Mol Biol Cell, 19, 65-77.

Snigdha S, Smith ED, Prieto GA, et al (2012). Caspase-3 activation as a bifurcation point between plasticity and cell death. Neurosci Bull, 28, 14-24.

Song W, Jiang R, Zhao CM (2012). Role of integrin-linked kinase in multi-drug resistance of human gastric carcinoma SGC7901/DDP cells. Asian Pac J Cancer Prev, 13, 5619-25.

Sun M, Jiang R, Li JD, et al (2011). MED19 promotes proliferation and tumorigenesis of lung cancer. Mol Cell Biochem, 355, 27-33.

Vojnic E, Mourao A, Seizl M, et al (2011). Structure and VP16 binding of the Mediator Med25 activator interaction domain. Nat Struct Mol Biol, 18, 404-9.

Wang JS, Ji AF, Wan HJ, et al (2012). Gene silencing of $\beta$-catenin by RNAi inhibits proliferation of human esophageal cancer cells by inducing G0/G1 cell cycle arrest. Asian Pac J Cancer Prev, 13, 2527-32.

Wang T, Hao L, Feng Y, et al (2011). Knockdown of MED19 by lentivirus-mediated shRNA in human osteosarcoma cells inhibits cell proliferation by inducing cell cycle arrest in the G0/G1 phase. Oncol Res, 19, 193-201.

Wen H, Feng CC, Ding GX, et al (2013). Med19 promotes bone metastasis and invasiveness of bladder urothelial carcinoma via bone morphogenetic protein 2. Ann Diagn Pathol, 17, 259-64.

Xu LL, Guo SL, Ma SR, et al (2012). Mammalian mediator 19 mediates H1299 lung adenocarcinoma cell clone conformation, growth, and metastasis. Asian Pac J Cancer Prev, 13, 3695-700.

Xu Y, Li C, Tian Q, et al (2012). Suppression of lung cancer metastasis-related protein 1 promotes apoptosis in lung cancer cells. Int J Mol Med, 30, 1481-6.

Yu HG, Wei W, Xia LH, et al (2013). FBW7 upregulation enhances cisplatin cytotoxicity in non-small cell lung cancer cells. Asian Pac J Cancer Prev, 14, 6321-6.

Yu W,Zhang Z, Min D, et al (2014). Mediator of RNA polymerase II transcription subunit 19 promotes osteosarcoma growth and metastasis and associates with prognosis. Eur J Cancer, 50, 1125-36.

Zhang H, Jiang H, Wang W, et al (2012). Expression of Med19 in bladder cancer tissues and its role on bladder cancer cell growth. Urol Oncol, 30, 920-7.

Zhang HQ, He B, Fang N, et al (2013). Autophagy inhibition sensitizes cisplatin cytotoxicity in human gastric cancer cell line SGC7901. Asian Pac J Cancer Prev, 14, 4685-8.

Zhu LJ, Yan WX, Chen ZW, et al (2013). Disruption of Mediator complex subunit 19 (Med19) inhibits cell growth and migration in tongue cancer. World J Surg Oncol, 11, 116.
Zou SW, Ai KX, Wang ZG, et al (2011). The role of Med19 in the proliferation and tumorigenesis of human hepatocellular carcinoma cells. Acta Pharmacol Sin, 32, 354-60. 